\title{
La fijación de metas de inflación da buenos resultados en América Latina
}

\author{
José García-Solanes y Fernando Torrejón-Flores
} inflación en cinco países de América Latina entre 2000 y 2007. Se realizan pruebas econométricas cuyos resultados revelan que la estrategia de metas inflacionarias ha contribuido a reducir el nivel y la variabilidad de las tasas de inflación y de interés a corto plazo, en contraste con países latinoamericanos que no adoptaron tales objetivos. Además, se constata empíricamente que el mantenimiento de esas metas ha contribuido a disminuir la variabilidad del crecimiento del PIB, si bien su efecto neto en el crecimiento económico aún no queda claro. La principal innovación técnica de este artículo consiste en la estimación de un modelo de efectos de tratamiento para solucionar el problema de endogeneidad vinculado a la adopción de objetivos de inflación, inherente a la mayoría de las pruebas econométricas realizadas hasta hoy en ese terreno.

PALABRAS CLAVE

CLASIFICACIÓN JEL
Inflación, política monetaria, macroeconomía, modelos econométricos, crecimiento económico, indicadores económicos, América Latina, Brasil, Chile, Colombia, México, Perú

E52, f21, f33

José García-Solanes. Facultad de Economía y Empresa, Universidad de Murcia, España. solanes@um.es Fernando Torrejón-Flores. Facultad de Ciencias Jurídicas y de la Empresa, Universidad Católica San Antonio de Murcia, España. ftorrejon@pdi.ucam.edu 


\section{I}

\section{Introducción}

En muchos estudios empíricos se demuestra que las economías de mercado emergentes que adoptaron regímenes monetarios de objetivos explícitos de inflación han logrado reducir sus tasas de inflación. Calderón y Schmidt-Hebbel (2003a y 2003b) mostraron que aquellos países de América Latina y el Caribe donde se fijaron metas de inflación (en lo sucesivo, MI) han sido capaces de disminuir sistemáticamente tanto sus tasas de inflación, como las desviaciones de estas con respecto a las metas establecidas. Lin y Ye (2009) obtuvieron un resultado similar a partir de varios métodos de PSM (propensity score matching) para 13 países en desarrollo. Corbo, Landerrechte y Schmidt-Hebbel (2002), por una parte, y Gonçalves y Salles (2008), por otra, encontraron que, en comparación con las economías que no habían establecido objetivos de inflación, los países en desarrollo que sí lo habían hecho consiguieron reducir tanto la inflación como la volatilidad del crecimiento económico. Valdés (2007) observó resultados similares para el caso de Chile. Batini y Laxton (2007) y el Fondo Monetario Internacional (FMI, 2005 y 2006) encontraron mayores ventajas macroeconómicas, concretadas en una inflación menor y una volatilidad más reducida en las tasas de inflación y de interés, los tipos de cambio, las reservas internacionales y el crecimiento económico con respecto a los países que no adoptaron ningún sistema de MI. Por último, también Brito y Bystedt (2010) indican que con ese régimen de política monetaria se reducen la tasa de inflación y su volatilidad, así como la tasa de crecimiento en las economías de mercado emergentes, aunque los resultados son menos significativos que los logrados en estudios anteriores.

Los resultados que, en última instancia, se alcancen con un régimen de MI dependerán mucho de la capacidad

\footnotetext{
$\square$ Los autores agradecen a Arielle Beyaert, Enrique Alberola y Josep Lluís Carrión-i-Silvestre sus comentarios acerca de la metodología econométrica aplicada en este artículo. Agradecen también a los participantes en los seminarios de la novena Conferencia anual sobre integración monetaria y económica en Europa (INFER, por sus siglas en inglés), celebrada en la Universidad de Loughborough en octubre de 2007; de la quinta edición de las Jornadas sobre Integración Económica, organizada por la Universitat Jaume I en noviembre de 2007, y de la undécima Jornada de Economía Internacional celebrada en la Universidad de Barcelona en junio de 2009. Todos los errores son de responsabilidad de los autores.
}

del esquema para fortalecer la credibilidad y rebajar las expectativas de inflación de los países en desarrollo ${ }^{1}$. Con relación a este aspecto, los defensores del sistema de MI presentan una visión optimista, basados en que la credibilidad potencial es elevada por el hecho de que los países en desarrollo suelen empezar a implantar ese régimen con instituciones muy débiles. Por eso, Svensson (1997); Mishkin (1999); King (2005); FMI (2006); y Blejer y otros (2001) aconsejaron la adopción de MI en economías de mercado emergentes durante los programas de ajuste estructural del FMI. Otros autores se manifestaron más desconfiados de este esquema de política monetaria, afirmando que existe una especie de "pecado original" y de obstáculos estructurales en la aplicación de políticas económicas, que impiden que los bancos centrales de estas economías aminoren sistemáticamente las expectativas de inflación (Calvo y Mishkin, 2003; Sims, 2005; Blanchard, 2005).

El análisis empírico de los efectos macroeconómicos del régimen de MI exige que se corrija el problema de endogeneidad. Hasta donde se sabe, el estudio de Brito y Bystedt (2010) es el único en que se aborda esta cuestión de forma explícita y rigurosa en el contexto de las economías de mercado emergentes. Estos autores analizaron, para el período 1980-2006, las repercusiones de la adopción del esquema de MI en las tasas de inflación y de crecimiento de 13 países en desarrollo. En el análisis utilizaron como referencia varios grupos de control sobre la base de una muestra de 33 economías emergentes sin objetivos explícitos de inflación. Aplicaron el estimador de datos de panel según el método generalizado de momentos simulados

\footnotetext{
${ }^{1}$ En la bibliografía se refleja un debate similar con respecto a la idoneidad de los regímenes de MI en economías en transición. Por otra parte, Jonas y Mishkin (2005) y Hrncir y Smidkova (2003), entre otros, consideran que - si bien los bancos centrales que mantienen objetivos directos de inflación se enfrentan en estos países a dificultades específicas (por ejemplo, predecir la inflación en un contexto de mayor incertidumbre y frecuentes turbulencias) - estos esquemas aún podrían brindar ventajas significativas, como un mayor control de las expectativas y menor flexibilidad de la política monetaria a corto plazo. En cambio, Kvasnicka (2000) estima que los regímenes de MI no resultan aconsejables para las economías en transición, muy sensibles a las incertidumbres y la inestabilidad en el mecanismo de transmisión de la política monetaria. Yigit (2010), tomando como marco de referencia a ocho países desarrollados, mostró la eficacia de las MI para reducir la inercia en las expectativas de inflación...
} 
(s-GMM) en dos etapas. Sus conclusiones apuntan a que las economías de mercado emergentes con estrategias de MI han logrado reducir la inflación, pero a costa de entorpecer el crecimiento económico.

En este artículo se aborda el problema de endogeneidad a través de la estimación (tras aplicar dos contrastes de regresión convencionales) de un modelo de efectos de tratamiento, según el procedimiento sugerido por Heckman (1979), Maddala (1983) y Greene (2003). Este método resulta especialmente indicado para examinar la repercusión de las variables cualitativas - como la adopción de MI- en variables cuantitativas. Consiste en estimar secuencialmente: i) una ecuación por el método probit orientada a evaluar hasta qué punto algunas variables extraídas de la teoría y de la literatura empírica afectan a la probabilidad de adoptar un régimen de MI, y ii) varias ecuaciones de respuesta, donde la variable dependiente es función de algunas variables macroeconómicas principales, entre ellas la adopción de MI como variable clave.

En este trabajo el análisis se centra en cinco países de América Latina que, a finales de 2007, aplicaban sistemas de MI, en comparación con otro grupo de 10 economías latinoamericanas donde no se había seguido ese régimen, observando el período comprendido entre 1980 y 2007. Por diversos motivos, América Latina proporciona un estudio de caso interesante para evaluar los efectos del esquema de MI en economías de mercado emergentes. En primer lugar, los cinco países latinoamericanos elegidos han sido precursores mundiales de la implementación de MI entre las economías de mercado emergentes. En segundo lugar, la elección de un panel compuesto únicamente por países de América Latina permite minimizar las diferencias económicas e institucionales entre los miembros del panel; ello contribuye a aislar mejor los efectos de la adopción de MI. De hecho, el estudio se centra en un grupo más homogéneo de economías que los que normalmente se consideran en la bibliografía.

Cabe destacar que el empleo de la metodología de efectos de tratamiento no está sujeto a distorsión por un sesgo temporal, ya que todos los países del grupo empezaron a aplicar sistemas de MI en torno del año 2000, que marca también la fecha de inicio en el presente análisis de efectos de tratamiento. Las pequeñas diferencias entre las fechas de adopción de objetivos de inflación no pueden afectar significativamente a los resultados. Además, no hay evidencias de que los países de América Latina incluidos en la muestra se hayan visto afectados por perturbaciones temporales muy diferentes desde el año 2000. También existen motivos para creer que la metodología empleada en este trabajo resulta adecuada para resolver el problema de la autoselección, dado que las variables que condujeron a cada país a aplicar el sistema de MI están determinadas endógenamente en el modelo que aquí se presenta. Así, se puede afirmar que la metodología econométrica de efectos de tratamiento contribuye a mejorar las estimaciones de los efectos de la adopción de MI en las variables macroeconómicas seleccionadas, en comparación con otras metodologías que no resuelven bien el problema de la autoselección ni el sesgo temporal.

El artículo se divide en cuatro secciones. Después de esta Introducción, en la sección II se realiza un análisis descriptivo con todas las variables relevantes (tasa de inflación, tasa de interés de corto plazo, y crecimiento económico) de los dos grupos de países, a fin de captar intuitivamente el efecto de las MI. En la sección III se aplican tres contrastes econométricos distintos para investigar con rigor la repercusión de la fijación de MI en el nivel y la variabilidad de la tasa de inflación, la tasa de interés a corto plazo y el crecimiento del producto interno bruto (PIB). Por último, en la sección IV se resumen las principales conclusiones empíricas.

Los resultados que se obtienen del análisis empírico pueden recapitularse como se indica a continuación. Tanto las pruebas de regresión como las de efectos de tratamiento señalan que los regímenes de MI han contribuido decisivamente a reducir tanto los niveles como la variabilidad de la inflación y las tasas de interés a corto plazo, así como a disminuir la variabilidad del crecimiento del PIB en comparación con otros regímenes monetarios durante el período objeto de estudio. Aunque el efecto final en el crecimiento del PIB no se puede discernir claramente - lo que no es extraño, considerando que el período observado es relativamente corto-, los resultados denotan que con el sistema de MI se ha mejorado el desempeño macroeconómico en comparación con otros regímenes de política monetaria que no incluyen una referencia explícita para anclar las expectativas de inflación. 


\section{II}

\section{Análisis descriptivo}

En esta sección se aplica estadística descriptiva para tener una primera impresión de los efectos macroeconómicos que podrían derivarse de la adopción de MI en cinco países de América Latina: Brasil, Chile, Colombia, México y Perú. La metodología general consiste en lo siguiente: i) para cada país que aplica MI, se comparan los resultados relativos a algunas variables macroeconómicas relevantes durante el período previo a la implementación del régimen con los del período posterior a su entrada en vigencia, y ii) se comparan los resultados (a los que remite el mismo conjunto de variables) que los países de cada grupo (con y sin objetivos de inflación) obtuvieron durante el lapso posterior a la aplicación de ese régimen. El grupo de economías sin MI tomado como referencia consta de 10 países: Argentina, Bolivia (Estado Plurinacional de), Costa Rica, Ecuador, El Salvador, Panamá, Paraguay, República Dominicana, Uruguay y
Venezuela (República Bolivariana de). Se han seleccionado dos grupos de países de América Latina, a efectos de comparar áreas que no solo comparten un contexto geográfico análogo, sino también grados de desarrollo económico e institucional similares.

Como paso previo, resulta útil describir los regímenes de tipo de cambio vigentes en los países de la muestra a lo largo del período de análisis. Las 15 economías seleccionadas no solo presentan una amplia gama de regímenes de tipo de cambio, sino también mecanismos monetarios muy diversos. En el cuadro 1 se observan los regímenes de tipo de cambio declarados por estos países al FMI en tres años distintos: 1985, 2002 y 2007. Como puede verse, la tendencia general muestra una transición desde esquemas intermedios hasta soluciones extremas: mientras que en 1985 una docena de los 15 regímenes de tipo de cambio correspondían a sistemas

CUADRO 1

América Latina: regímenes de tipo de cambio, 1985, 2002 y 2007

\begin{tabular}{|c|c|c|c|}
\hline \multirow{2}{*}{ Países con metas de inflación } & 1985 & 2002 & 2007 \\
\hline & \multicolumn{3}{|c|}{ Regímenes de tipo de cambio } \\
\hline Brasil & Intermedio & Flotación & Flotación (FI) \\
\hline Chile & Intermedio & Flotación & Flotación (FI) \\
\hline Colombia & Intermedio & Flotación & Flotación (FC) \\
\hline México & Intermedio & Flotación & Flotación (FI) \\
\hline Perú & Intermedio & Flotación & Flotación (FC) \\
\hline Países sin metas de inflación & \multicolumn{3}{|c|}{ Regímenes de tipo de cambio } \\
\hline Argentina & Intermedio & Flotación & Flotación (FC) \\
\hline Bolivia (Estado Plurinacional de) & Flotación & Intermedio & Intermedio (PM) \\
\hline Costa Rica & Intermedio & Intermedio & Intermedio (PM) \\
\hline República Dominicana & Intermedio & Intermedio & Flotación (FC) \\
\hline Ecuador & Intermedio & Paridad fija & Paridad fija (SMN) \\
\hline El Salvador & Intermedio & Paridad fija & Paridad fija (SMN) \\
\hline Panamá & Paridad fija & Paridad fija & Paridad fija (SMN) \\
\hline Paraguay & Intermedio & Flotación & Flotación (FC) \\
\hline Uruguay & Flotación & Flotación & Flotación (FC) \\
\hline Venezuela (República Bolivariana de) & Intermedio & Flotación & Paridad fija (PC) \\
\hline
\end{tabular}

Fuente: A. Berg, E. Borensztein y P. Mauro, “An evaluation of monetary regime options for Latin America”, IMF Working Paper, N 02/211, Washington, D.C., Fondo Monetario Internacional, 2002; y Fondo Monetario Internacional (FMI), "De Facto Classification of Exchange Rate Regimes and Monetary Policy Frameworks", abril de 2008 [en línea] http://www.imf.org/external/np/mfd/er/2008/eng/0408.htm.

Notas

PM: paridad móvil (tipo de cambio móvil); FI: flotación independiente; FC: flotación controlada sin trayectoria previamente anunciada sobre el tipo de cambio; SMN: sin moneda nacional de curso legal; PC: paridad fija convencional. 
intermedios, en 2007 solo quedaban dos. Según el estudio empírico de Calderón y Schmidt-Hebbel (2003b), la ruptura estructural se produjo en 1998, inmediatamente después de la crisis asiática.

De los nueve países que dejaban flotar su moneda en 2007, tres operaban con un tipo de cambio flexible e independiente, y los otros seis presentaban flotaciones controladas sin una trayectoria de tipo de cambio previamente anunciada. Los tres bancos centrales que permitían una flotación independiente pertenecen al grupo de países que adoptaron regímenes de MI y no recurrieron a intervenciones sistemáticas para amortiguar las fluctuaciones de su tipo de cambio.

\section{Fijación de metas de inflación en América Latina}

Hasta la fecha, cinco países de América Latina han implementado estrategias de MI más o menos decididas. Mishkin y Savastano (2002) ofrecen un análisis detallado de las características de estos sistemas hasta el año 2001. En el cuadro 2 se aprecian las principales características de los regímenes de MI aplicados en los cinco países. Las fechas de inicio que figuran entre paréntesis indican cuándo empezó cada banco central a publicar informes de inflación.

En enero de 1991, Chile se convertía en el primer país que otorgaba independencia a su banco central y declaraba que la estabilidad de los precios era uno de sus principales objetivos. La consecuencia fue una reducción gradual de la inflación interna. Sin embargo, hasta 1999 el banco central no anunció explícitamente un objetivo de inflación plurianual, y en mayo de 2000 empezó a publicar informes de inflación que incluían sus previsiones de inflación de referencia. La consistencia de las finanzas públicas y la solvencia del sistema financiero han sido dos características clave de la economía chilena en que se ha apoyado la implantación sin restricciones de un sistema de MI.

En 1999, Brasil adoptó un régimen de política monetaria que incorporaba los principales ingredientes de un esquema de MI. El banco central publicó acto seguido un exhaustivo informe de inflación. Desde entonces ha ido ganando en independencia, de acuerdo con la voluntad institucional de garantizar el éxito del sistema de MI, sin perder de vista, además, que los déficits fiscales debían volver a niveles que impidieran cualquier posibilidad de predominio fiscal.

En 1991, el Banco Central de Colombia empezó a anunciar metas numéricas explícitas con respecto a la tasa de inflación anual. Esta estrategia antiinflacionista no surtió efecto hasta 1999, debido a que el banco central siguió dando prioridad a otros objetivos, sobre todo a la estabilización de la producción y la competitividad externa en la medida en que se vieran amenazadas por

CUADRO 2

América Latina (cinco países): regímenes de fijación de objetivos directos de inflación por país

\begin{tabular}{|c|c|}
\hline País & Fecha de inicio y características principales \\
\hline Brasil & $\begin{array}{l}\text { Fecha de inicio: junio de } 1999 \text { (septiembre de 1999). } \\
\text { Metas de inflación: } 8 \% \pm 2 \% \text { (1999); } 6 \% \pm 2 \% \text { (2000); } 4 \% \pm 2 \% \text { (2006-2007). } \\
\text { Informe de inflación y anuncio de objetivos de inflación plurianuales. } \\
\text { Posición fiscal débil y sistema financiero relativamente sólido. }\end{array}$ \\
\hline Chile & $\begin{array}{l}\text { Fecha de inicio: enero de } 1991 \text { (mayo de } 2000) \text {. } \\
\text { Metas de inflación: } 15 \%-20 \% \text { (1991); 3,5\% (2000); 2\%-4\% (2001-2007). } \\
\text { Informe de inflación y anuncio de objetivos plurianuales. } \\
\text { Posición fiscal sólida y sistema financiero relativamente sólido. }\end{array}$ \\
\hline Colombia & $\begin{array}{l}\text { Fecha de inicio: enero de } 1991 \text { (enero de 1999). } \\
\text { Metas de inflación: } 22 \%(1991) ; 10 \%(2000) ; 4,5 \% \pm 0,5 \% \text { (2006); 4\% } \pm 0,5 \% \text { (2007). } \\
\text { Informe de inflación y anuncio de objetivos plurianuales. } \\
\text { Posición fiscal sólida y sistema financiero relativamente sólido. }\end{array}$ \\
\hline México & $\begin{array}{l}\text { Fecha de inicio: enero de } 1996 \text { (abril de 2000). } \\
\text { Metas de inflación: } 20,5 \%(1996) ;<10 \%(2000) ; 3 \% \pm 1 \%(2003-2007) \text {. } \\
\text { Informe de inflación y anuncio de objetivos plurianuales. } \\
\text { Posición fiscal sólida y sistema financiero relativamente sólido actualmente. }\end{array}$ \\
\hline Perú & $\begin{array}{l}\text { Fecha de inicio: enero de } 1994 \text { (junio de 2002). } \\
\text { Metas de inflación: } 15 \%-20 \% \text { (1994); 3,5\%-4\% (2000); 2,5\% } 1 \% \text { (2002-2006); 2\% } 1 \% \text { (2007). } \\
\text { Informe de inflación y anuncio de objetivos plurianuales. } \\
\text { Posición fiscal débil pero sistema financiero relativamente sólido. }\end{array}$ \\
\hline
\end{tabular}

Fuente: elaboración propia sobre la base de los respectivos informes económicos de los bancos centrales de los cinco países. 
la meta de inflación. Por otra parte, hasta ese año no se había conseguido controlar suficientemente el déficit presupuestario. A partir de enero de 1999 se produjo un cambio positivo, cuando el Banco Central de Colombia empezó a publicar informes sobre inflación. Además, en septiembre de ese mismo año el tipo de cambio empezó a flotar libremente. Por último, el anuncio de objetivos de inflación plurianuales a partir de octubre de 2000 también ha contribuido al éxito del nuevo régimen en los últimos años.

El Banco de México esperó hasta contar con suficiente credibilidad en el control de la inflación para implantar un sistema de MI; fue en enero de 1999, cuando la tasa anual de inflación del $12,3 \%$ se encontraba por debajo del objetivo (fijado en el 13\%). En abril de 2000, el Banco de México comenzó a publicar un informe mensual de inflación.

Con el anuncio en 1994 de un esquema de MI, se abría en el Perú un fructífero período para el control inflacionista. La tasa de inflación se redujo drásticamente de más de un $20 \%$ en 1994 a un 3\% en 2001. Sin embargo, en ese tiempo las autoridades monetarias peruanas no lograron alcanzar la credibilidad necesaria, ya que su sistema monetario carecía de muchas de las características esenciales de un régimen de MI; por ejemplo, el anuncio de objetivos de inflación plurianuales, la publicación de informes de inflación, y los mecanismos para otorgar responsabilidades al Banco Central de Reserva del Perú. En junio de 2002, esa situación se había corregido casi completamente y la política monetaria empezó a cumplir los principales requisitos de un auténtico sistema de MI.

\section{Resultados macroeconómicos}

Para sopesar en qué medida un esquema de MI puede haber contribuido a mejorar los resultados macroeconómicos en los cinco países de América Latina que siguieron ese esquema, se investigaron los resultados con respecto a la inflación, las tasas de interés de los depósitos bancarios y el crecimiento del PIB. Se aplicaron cálculos descriptivos tanto para las variables de las series cronológicas como para las variables transversales de los dos grupos de países.

\section{a) Análisis descriptivo de las variables de las series cronológicas}

Se compiló una base de datos con información mensual referente a un período relativamente largo (entre enero de 1981 y diciembre de 2007) y se calcularon las tasas anualizadas para las tres variables relevantes: inflación, tasa de interés a corto plazo (depósitos bancarios con vencimiento a un año) y crecimiento del PIB. Debido a limitaciones de disponibilidad de datos, las tasas de crecimiento del PIB se calcularon a partir de observaciones trimestrales. La muestra se divide en dos períodos en cada país, teniendo en cuenta la entrada en vigor del régimen de objetivos explícitos de inflación. La fecha de inicio de aplicación del régimen corresponde al momento en que el banco central empezó a publicar informes de inflación. Así, los cinco países que implantaron estos sistemas de MI lo hicieron entre enero de 1999 y junio de 2002 (véase el cuadro 3$)^{2}$.

Los datos relativos a la longitud de cada submuestra se presentan en el cuadro 3. En la muestra 1, correspondiente al período anterior a la aplicación del régimen de MI, no se incluyen los años de hiperinflación en los respectivos países. La muestra 2 abarca el período posterior a la adopción de MI. Se consideran como hiperinflación las tasas de inflación que sobrepasan el percentil 95 en la distribución de inflación de la muestra de todos los países de América Latina.

En el cuadro 4 se señalan el promedio y la desviación típica de las tasas mensuales de inflación a lo largo de 12 meses (sobre una base anual) para cada submuestra y país. Con respecto a los niveles, se constata que la tasa de inflación se redujo drásticamente entre el primer y el segundo subperíodos en cada país. El Brasil consiguió los mejores resultados, incluso si no se tienen en cuenta las elevadísimas cifras de los años en que el país experimentó hiperinflación. En promedio, durante el segundo período la tasa de inflación se redujo aproximadamente hasta un 9\% del valor registrado en el período de la primera muestra.

En cuanto a la variabilidad de la tasa de inflación, las columnas tercera y cuarta del cuadro 4 ilustran reducciones de orden similar a las registradas en los niveles de inflación en cada país. El descenso de la variabilidad es especialmente pronunciado en los países que presentan una inflación inicial mayor. En resumen, la disminución de la inflación es muy significativa en todos los países y se ha evidenciado tanto en los niveles como en la variabilidad.

En el cuadro 5 se proporciona información similar a la del cuadro 4, pero en referencia a la tasa de interés nominal anualizada para depósitos bancarios. Como puede

\footnotetext{
2 Otros autores argumentan que la implantación sin trabas de estos regímenes se produjo tras varios intentos. No todos ellos coinciden en las fechas de inicio sugeridas, si bien las diferencias son relativamente poco significativas. Así, de acuerdo con Mishkin y Schmidt-Hebbel (2007), el "período de objetivos fijos" (stationary target period) se inicia entre enero de 2001 y enero de 2003, mientras que Batini y Laxton (2007) consideran que el inicio fue entre el segundo trimestre de 1999 y el primero de 2002.
} 
CUADRO 3

\section{América Latina (cinco países): submuestras por país}

\begin{tabular}{|c|c|c|c|c|}
\hline País & $\begin{array}{l}\text { Muestra 1: } \\
\text { antes de adoptar } \\
\text { metas de inflación }\end{array}$ & $\begin{array}{c}\text { Período de } \\
\text { hiperinflación }\end{array}$ & $\begin{array}{c}\text { Inicio de régimen } \\
\text { de metas de inflación }\end{array}$ & $\begin{array}{l}\text { Muestra 2: } \\
\text { luego de adoptar } \\
\text { metas de inflación }\end{array}$ \\
\hline Brasil & 1981: ene-1999: ago & 1987: feb-1995: mar & 1999: sep & 1999: sep-2007: dic \\
\hline Chile & 1981: ene-2000: abr & & 2000: may & 2000: may-2007: dic \\
\hline Colombia & 1981: ene-1998: dic & & 1999: ene & 1999: ene-2007: dic \\
\hline México & 1981: ene-2000: mar & & 2000: abr & 2000: abr -2007: dic \\
\hline
\end{tabular}

Fuente: elaboración propia sobre la base de los respectivos informes económicos de los bancos centrales de los cinco países.

Nota: la muestra 1 abarca los años anteriores a la adopción de metas de inflación. La muestra 2 abarca los años posteriores a la implantación de metas de inflación. En la muestra 1 no se incluyen los años de hiperinflación.

Se considera como hiperinflación al $5 \%$ de las observaciones con tasas de inflación mensual más elevadas.

CUADRO 4

\section{América Latina (cinco países): inflación por país \\ (Observaciones mensuales anualizadas, en porcentajes)}

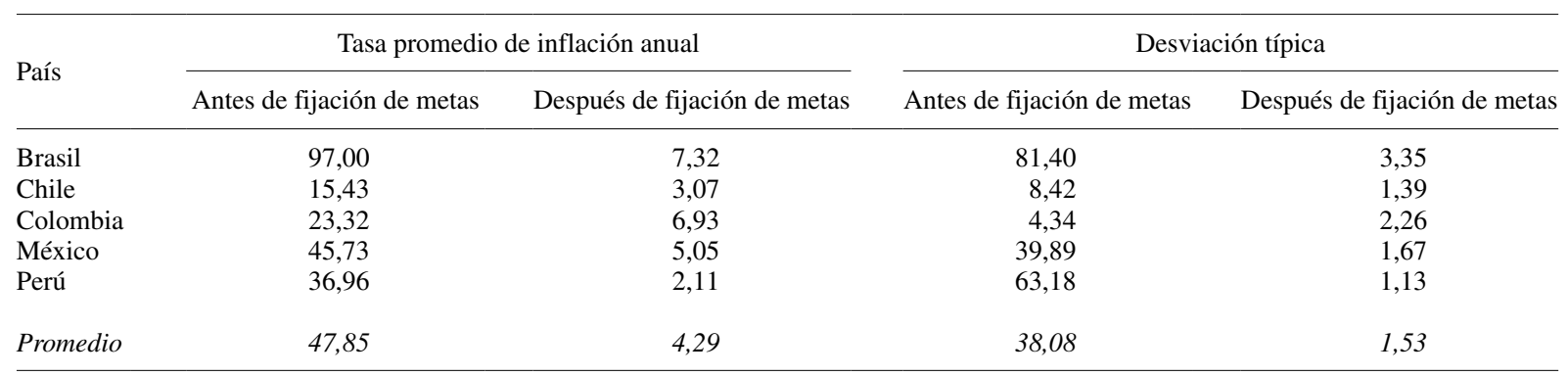

Fuente: elaboración propia sobre la base de datos de Estadísticas Financieras Internacionales del Fondo Monetario Internacional.

CUADRO 5

América Latina (cinco países): tasa de interés anualizada de los depósitos bancarios (Observaciones mensuales anualizadas, en porcentajes)

\begin{tabular}{|c|c|c|c|c|}
\hline \multirow{2}{*}{ País } & \multicolumn{2}{|c|}{ Tasa promedio de interés } & \multicolumn{2}{|c|}{ Desviación típica } \\
\hline & Antes de fijación de metas & Después de fijación de metas & Antes de fijación de metas & Después de fijación de metas \\
\hline Chile & 23,02 & 4,57 & 13,87 & 2,06 \\
\hline Colombia & 31,16 & 10,19 & 4,35 & 4,82 \\
\hline México & 36,78 & 4,10 & 24,85 & 1,83 \\
\hline Promedio & 86,81 & 10,36 & 40,22 & 3,40 \\
\hline
\end{tabular}

Fuente: elaboración propia sobre la base de datos de Estadísticas Financieras Internacionales del Fondo Monetario Internacional.

apreciarse, tanto los promedios como las desviaciones típicas se reducen también sustancialmente en cada país. En los países que se incluyen en el análisis empírico, el sistema de MI contribuyó claramente a facilitar la política monetaria y a disminuir tensiones en los mercados monetario y crediticio dentro del ámbito nacional. Por lo tanto, no es de extrañar que el esquema de MI cree un entorno favorable para las decisiones de inversión, lo que a su vez podría contribuir a impulsar el crecimiento económico a mediano y largo plazo. 


\begin{tabular}{lccccc}
\hline \multirow{2}{*}{ País } & \multicolumn{2}{c}{ Tasa de crecimiento promedio del PIB } & & \multicolumn{2}{c}{ Desviación típica } \\
\cline { 2 - 3 } \cline { 5 - 6 } & Antes de fijación de metas & Después de fijación de metas & & Antes de fijación de metas & Después de fijación de metas \\
\hline Brasil & 2,02 & 3,18 & 4,31 & 2,45 & 1,64 \\
Chile & 6,28 & 2,93 & 3,66 & 1,26 \\
Colombia & 3,09 & 2,74 & 1,34 & 2,35 \\
México & 3,36 & 6,08 & 3,59 & 1,69 \\
Perú & 3,99 & 3,85 & 3,21 & 2,02 \\
Promedio & 3,75 & & 3,05 & \\
\hline
\end{tabular}

Fuente: elaboración propia sobre la base datos de la página web de estadísticas de la Comisión Económica para América Latina y el Caribe (CEPAL). Nota: las cifras corresponden a las tasas anuales de crecimiento del producto interno bruto (PIB), sobre la base de observaciones trimestrales. Muestra: del primer trimestre de 1992 al cuarto trimestre de 2007.

En el cuadro 6 se presentan datos referidos al crecimiento económico anualizado, calculados sobre la base de observaciones trimestrales del PIB real. Pareciera que la aplicación de un régimen de mI va acompañada de una manifiesta reducción de la volatilidad del crecimiento, aunque los efectos en el nivel de este son ambiguos, pues solo se observan en el Brasil y el Perú.

b) Análisis descriptivo de las variables transversales Para tener una perspectiva más amplia de los resultados de la aplicación del esquema de MI, se considera útil comparar el desempeño macroeconómico de dos grupos de economías de América Latina: uno, de países que han implementado un régimen de ese tipo y, otro, de países que no lo han hecho. El primer grupo consta de los cinco países considerados en la sección anterior, y el segundo grupo reúne a los 10 países siguientes: Argentina, Bolivia (Estado Plurinacional de), Costa Rica, Ecuador, El Salvador, Panamá, Paraguay, República Dominicana, Uruguay y Venezuela (República Bolivariana de). Para los dos grupos de países se calcularon valores transversales, mensuales para la inflación y la tasa de interés, y trimestrales para el crecimiento del PIB, referidos todos al período de enero de 2000 a diciembre de 2007.

En el gráfico 1 se presentan los resultados respecto de las tres variables y los dos grupos de países.

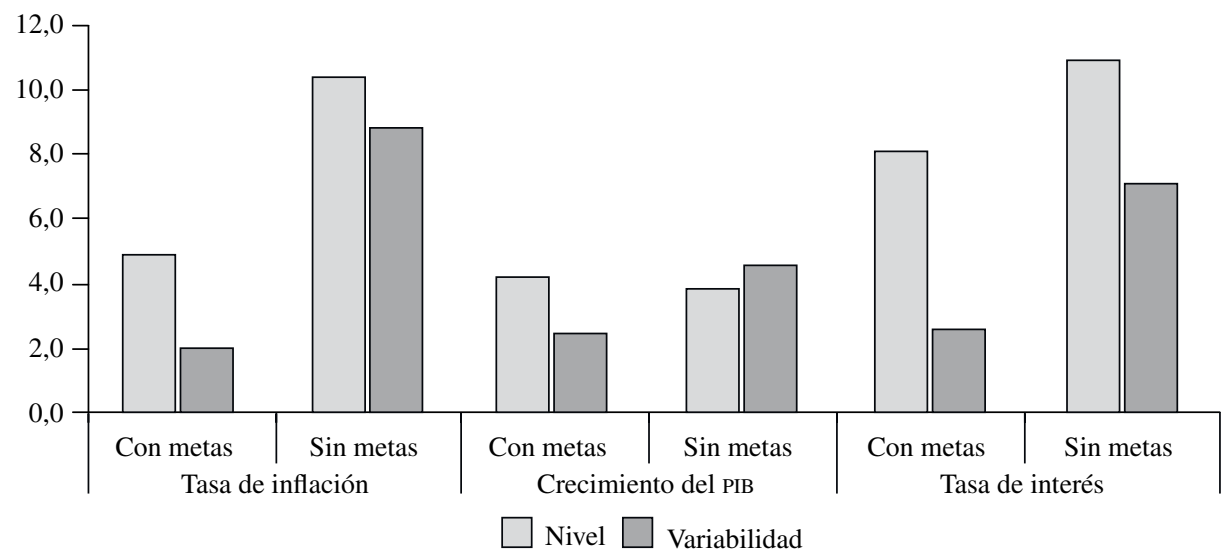

Fuente: elaboración propia sobre la base datos de la página web de estadísticas de la Comisión Económica para América Latina y el Caribe (CEPAL) y de la base de datos de Estadísticas Financieras Internacionales del Fondo Monetario Internacional. 
Como puede observarse, dichos resultados respaldan las conclusiones del análisis anterior sobre la base de variables cronológicas: en comparación con los países que no adoptaron MI durante el período de la muestra, los que sí lo hicieron obtuvieron: i) una tasa de inflación menor y menos volátil; ii) una menor variabilidad del crecimiento del PIB, y iii) niveles mucho más bajos y menos volátiles de las tasas de interés de corto plazo. Los efectos netos en el crecimiento promedio del PIB no quedan claros.

\section{III}

\section{Análisis de series cronológicas y de datos de panel}

\section{Regresiones de series cronológicas}

En esta sección se realizan regresiones con variables ficticias, según la metodología de Ball y Sheridan (2005), para evaluar si los niveles y la variabilidad de tres variables (la tasa de inflación, la tasa de interés a corto plazo y el crecimiento del PIB) han cambiado significativamente entre los períodos previos y posteriores a la fijación de MI en los respectivos países.

Para ello se estima la siguiente ecuación:

$$
x_{i, t}=\alpha_{i}+\delta_{i} F_{i, t}+\gamma_{i} x_{L A T, t}+\theta_{i} G B_{i, t}+\varepsilon_{i, t}
$$

donde $x_{i, t}$ es la variable analizada del país $i$ correspondiente al período $t, F_{i, t}$ y es una variable ficticia que toma el valor 1 durante los períodos en que el país $i$ aplica metas de inflación y el valor 0 durante el resto de la muestra. El parámetro $\delta_{i}$ mide el efecto del esquema de MI en la variable $x_{i, t}$.

En la ecuación (1) se incluyen dos variables de control para aislar el efecto de la adopción de MI. La primera, $x_{L A T, t}$, se refiere al promedio de $x_{i, t}$ de un gran grupo de países de América Latina. Se incluye para evitar las estimaciones sesgadas del parámetro $\delta_{i}$ que se producen cuando la variable $x_{i, t}$ converge hacia una media común en la muestra de países. Por ejemplo, si $x_{i, t}$ es la tasa de inflación de Chile, la reducción del valor de esta variable observada entre los períodos anterior y posterior a la fijación de MI podría deberse a una tendencia general del grupo de países y no a la implantación de dichas metas. La segunda variable de control, $G B_{i, t}$, registra la fortaleza del presupuesto público y se mide por el cociente entre el ingreso y el gasto del gobierno central.

Ball y Sheridan (2005) siguieron una metodología similar para analizar los efectos de la implantación de
MI en un grupo de países industrializados, aunque sin realizar estimaciones específicas para cada país.

Primero se estima la ecuación (1) aplicada al nivel y a la desviación típica de la tasa de inflación. La inflación se calcula mediante la variación anual del índice de precios al consumidor (IPC), y su variabilidad se mide con la desviación típica de esa variable. A fin de determinar con más precisión la incidencia de las MI en estas variables, se realizan regresiones para tres muestras que se diferencian por la fecha de inicio del período anterior a la fijación de MI. El primero empieza en enero de 1981, el segundo en enero de 1992, y el tercero en enero de 1996. Si fuera cierto que con la implantación de MI cambia significativamente la evolución de las variables (de modo similar a un cambio estructural), cabría esperar que los efectos de la fijación de objetivos explícitos de inflación fueran mayores y más significativos estadísticamente cuanto más largos fuesen los subperíodos anteriores a la adopción de este esquema de política monetaria.

En el cuadro 7 se muestran los resultados de regresión para la tasa de inflación de cada país con MI. En las dos primeras columnas aparece la estimación de $\delta_{i}$ referida al nivel y la desviación típica de la inflación para la muestra más larga, que va de enero de 1981 a diciembre de 2007. En la estimación no se incluye la variable de control $G B_{i, t}$ debido a la ausencia de observaciones mensuales antes de 1992. Según los valores estimados de los parámetros y de los estadísticos $t$, el establecimiento de MI ha resultado muy eficaz para disminuir tanto la tasa de inflación como su variabilidad en cada país. Por ejemplo, en el caso de Chile, los valores $\delta_{\text {Chile }}=-6,83$ y $\delta_{\text {Chile }}=-1,36$ indican que la tasa de inflación y su variabilidad disminuyeron, en promedio, 6,83 y 1,36 puntos porcentuales, respectivamente, tras la adopción del esquema. Los estadísticos $t(-8,19$ y 
América Latina (cinco países): tasa de inflación, tres períodos

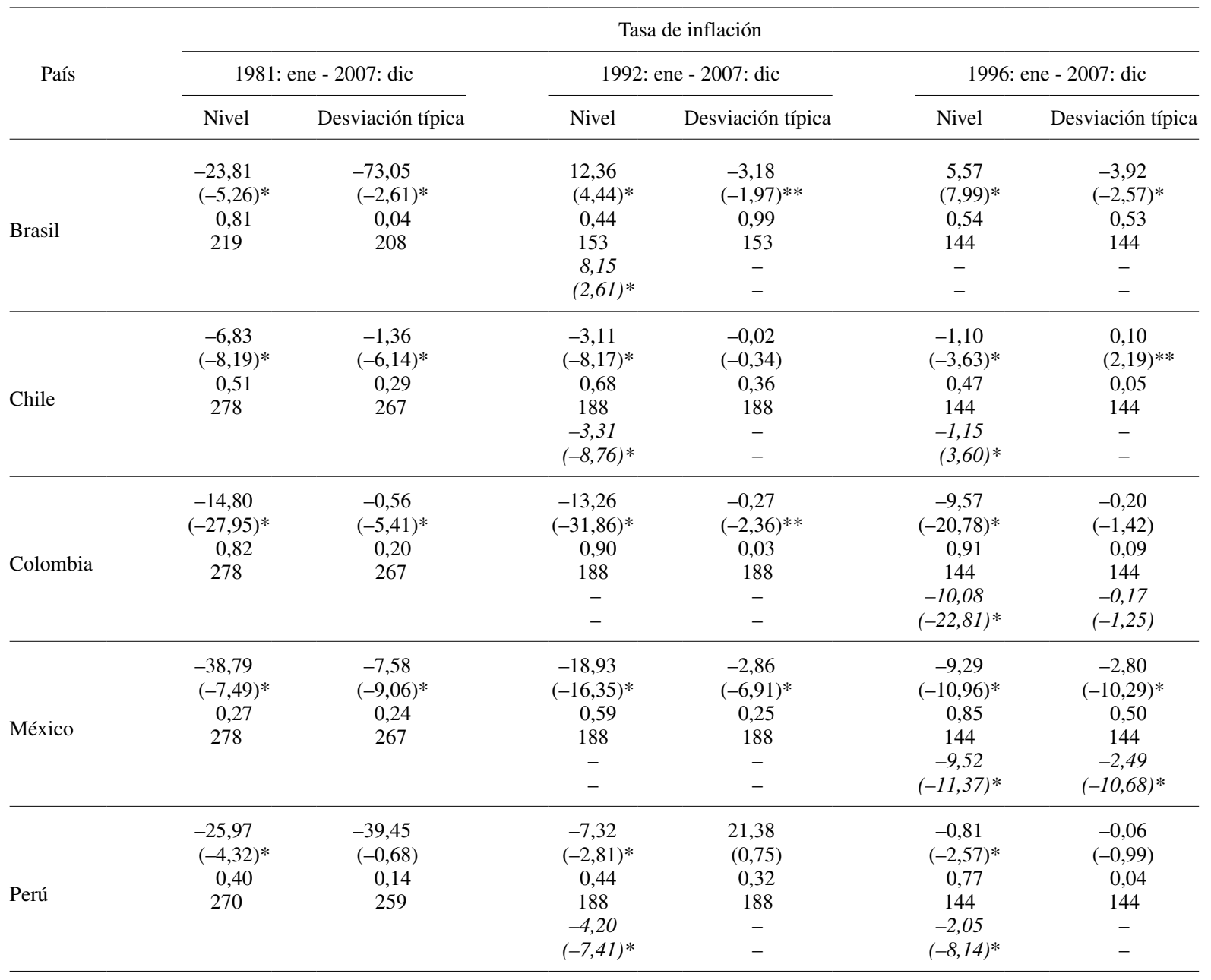

Fuente: Base de datos de Estadísticas Financieras Internacionales del Fondo Monetario Internacional.

Datos mensuales para cada país:

- En la primera fila se muestra el valor estimado de $\delta_{i}$.

- En la segunda fila se muestran los estadísticos $t$ según la hipótesis nula $H_{0}: \delta_{i}=0$.

Los niveles de significatividad son: $1 \%(*)$ y $5 \%(* *)$, respectivamente.

- En la tercera y cuarta filas se muestran los datos estadísticos $\bar{R}^{2}$ y el número de observaciones, respectivamente.

- Las celdas que contienen cifras en cursiva en la quinta y sexta filas corresponden a casos en que el presupuesto gubernamental es estadísticamente significativo. En la quinta fila se muestra el valor estimado de $\delta_{i}$ y en la sexta fila, sus estadísticos $t$. La ausencia de resultados para esta estimación indica que el presupuesto gubernamental no es estadísticamente significativo.

-6,14) permiten rechazar claramente la hipótesis nula de ausencia de significatividad en cada una de estas estimaciones.

En las columnas tercera a sexta del cuadro 7 se registran los resultados para los dos períodos más cortos con la variable $G B_{i, t}$ en las regresiones. Cuando $G B_{i, t}$ es estadísticamente significativa, el valor estimado de $\delta_{i}$ y su estadístico $t$ se indican con números en cursiva en las filas quinta y sexta de los países correspondientes.
Con fines comparativos, en esos casos también se realizan las mismas regresiones excluyendo $G B_{i, t}$; los resultados aparecen en la primera y segunda filas de cada país. Según puede verse, excepto en los dos períodos más cortos de Brasil, $\delta_{i}$ siempre tiene un valor negativo y es estadísticamente significativo en las regresiones correspondientes al nivel de inflación, así como en la mayoría de las regresiones referidas a la variabilidad de la inflación. Además, al incluirse 
$G B_{i, t}$ suele mejorar la significatividad estadística del parámetro $\delta_{i}$.

En resumen, los resultados de las regresiones indican que la adopción de MI ha impulsado a la baja tanto el nivel como la variabilidad de la inflación.

En el cuadro 8 se muestran los resultados de las regresiones para la tasa de interés anualizada de los depósitos bancarios calculada a partir de observaciones mensuales. Debido a las limitaciones de disponibilidad de datos, las estimaciones se ciñen a los períodos más cortos, de enero de 1992 a diciembre de 2007 y de enero de 1996 a diciembre de 2007.

Se observa que con la fijación de MI se produjo una reducción significativa en el nivel y la variabilidad de

CUADRO 8

América Latina (cinco países): tasa de interés

\begin{tabular}{|c|c|c|c|c|}
\hline \multirow{3}{*}{ País } & \multicolumn{4}{|c|}{ Tasa de interés } \\
\hline & \multicolumn{2}{|c|}{ 1992: ene - 2007: dic } & \multicolumn{2}{|c|}{ 1996: ene - 2007: dic } \\
\hline & Nivel & Desviación típica & Nivel & Desviación típica \\
\hline \multirow{6}{*}{ Brasil } & $-3,00$ & 4,06 & $-4,81$ & $-4,10$ \\
\hline & $(-1,86)^{* * *}$ & $(1,93) * * *$ & $(-4,33)^{*}$ & $(-9,61)^{*}$ \\
\hline & 0,64 & 0,99 & 0,60 & 0,47 \\
\hline & 153 & 153 & 144 & 144 \\
\hline & $-6,90$ & - & $-6,19$ & - \\
\hline & $(-4,69)^{*}$ & - & $(-5,46)^{*}$ & - \\
\hline \multirow{6}{*}{ Chile } & $-8,50$ & $-3,52$ & $-5,88$ & $-2,37$ \\
\hline & $(-13,44)^{*}$ & $(-13,85)^{*}$ & $(-8,62)^{*}$ & $(-15,37)^{*}$ \\
\hline & 0,59 & 0,56 & 0,65 & 0,66 \\
\hline & 188 & 188 & 144 & 144 \\
\hline & - & $-3,35$ & $-5,15$ & $-2,39$ \\
\hline & - & $(-13,24) *$ & $(-7,85) *$ & $(-17,85)$ \\
\hline \multirow{6}{*}{ Colombia } & $-19,62$ & $-1,29$ & $-12,38$ & $-0,94$ \\
\hline & $(-26,75)^{*}$ & $(-4,76)^{*}$ & $(-12,01)^{*}$ & $(-3,06)^{*}$ \\
\hline & 0,81 & 0,13 & 0,84 & 0,23 \\
\hline & 188 & 188 & 144 & 144 \\
\hline & - & - & - & $-0,92$ \\
\hline & - & - & - & $(-3,06)^{*}$ \\
\hline \multirow{6}{*}{ México } & $-16,52$ & $-3,22$ & $-8,11$ & $-2,67$ \\
\hline & $(-15,34)^{*}$ & $(-8,31)^{*}$ & $(-9,30)^{*}$ & $(-10,90)^{*}$ \\
\hline & 0,56 & 0,32 & 0,78 & 0,51 \\
\hline & 188 & 188 & 144 & 144 \\
\hline & - & - & $-7,16$ & $-2,36$ \\
\hline & - & - & $(-9,62)$ & $(-10,33)$ \\
\hline \multirow{6}{*}{ Perú } & $-7,94$ & $-5,54$ & $-5,78$ & $-0,33$ \\
\hline & $(-7,96)^{*}$ & $(-2,53) * *$ & $(-15,76)^{*}$ & $(-5,19)^{*}$ \\
\hline & 0,36 & 0,05 & 0,83 & 0,27 \\
\hline & 188 & 188 & 144 & 144 \\
\hline & $-7,21$ & $-0,13$ & $-6,24$ & $-0,12$ \\
\hline & $(-24,26)^{*}$ & $(-1,88) * * *$ & $(-17,60) *$ & $(-1,88) * * *$ \\
\hline
\end{tabular}

Fuente: Base de datos de Estadísticas Financieras Internacionales del Fondo Monetario Internacional.

Datos mensuales para cada país:

- En la primera fila se muestra el valor estimado de $\delta_{i}$.

- En la segunda fila se muestran los estadísticos $t$ según la hipótesis de nulidad $H_{0}: \delta_{i}=0$.

Los niveles de significatividad son los siguientes: $1 \%(*), 5 \%(* *)$ y $10 \%(* * *)$, respectivamente.

- En la tercera y cuarta filas se muestran los datos estadísticos $\bar{R}^{2}$ y el número de observaciones, respectivamente.

- Las celdas que contienen números en cursiva en la quinta y sexta filas corresponden a casos en que el presupuesto gubernamental es estadísticamente significativo. En la quinta fila se muestra el valor estimado de $\delta_{i}$ y en la sexta fila, sus estadísticos $t$. La ausencia de resultados para esta estimación indica que el presupuesto gubernamental no es estadísticamente significativo. 
la tasa de interés nominal (aunque no ocurrió así con la variabilidad de las tasas de interés del Brasil durante el primer período). Como en el cuadro 7 , los resultados de las estimaciones con la inclusión de $G B_{i, t}$ (cifras en cursiva) se muestran solamente cuando el presupuesto del gobierno es estadísticamente significativo. En esos casos, la inclusión de $G B_{i, t}$ mejora claramente la calidad estadística de los resultados.

En el cuadro 9 se presentan los resultados de la regresión para el crecimiento del PIB con observaciones trimestrales. Las estimaciones se limitan a las dos muestras más cortas de acuerdo con la información disponible. Dado que la inclusión de $G B_{i, t}$ dio lugar a estimaciones estadísticamente no significativas, los resultados correspondientes no figuran en el cuadro 9. Conviene resaltar que con la aplicación del esquema de MI, en la mayoría de los casos se aminora significativamente la variabilidad del crecimiento del PIB. Sin embargo, los resultados relativos al crecimiento son menos concluyentes. En algunos casos, el efecto es positivo (por ejemplo, para el Brasil y el Perú durante el período 1996-2007), pero para la mayoría de los países no es significativo, o incluso es negativo (Chile, 1992-2007; México, 1996-2007).

\section{Regresiones con datos de panel}

En esta subsección se realizan regresiones de panel que incluyen una variable ficticia para captar las diferencias macroeconómicas entre los países que han fijado MI y los que no lo han hecho.

CUADRO 9

América Latina (cinco países): crecimiento del PIB

\begin{tabular}{|c|c|c|c|c|}
\hline \multirow{3}{*}{ País } & \multicolumn{4}{|c|}{ Crecimiento del PIB } \\
\hline & \multicolumn{2}{|c|}{ 1er. trim. $1992-4^{\circ}$ trim. 2007} & \multicolumn{2}{|c|}{ 1er. trim. $1996-4^{\circ}$ trim. 2007} \\
\hline & Nivel & Desviación típica & Nivel & Desviación típica \\
\hline Brasil & $\begin{array}{c}0,59 \\
(1,37) \\
0,34 \\
61\end{array}$ & $\begin{array}{c}-0,27 * * \\
(-2,02) \\
0,05 \\
58\end{array}$ & $\begin{array}{c}1,51 * \\
(4,26) \\
0,59 \\
48\end{array}$ & $\begin{array}{c}-0,25 \\
(-1,57) \\
0,04 \\
48\end{array}$ \\
\hline Chile & $\begin{array}{c}-1,86^{*} \\
(-3,23) \\
0,39 \\
61\end{array}$ & $\begin{array}{c}-0,46^{*} \\
(-3,10) \\
0,25 \\
58\end{array}$ & $\begin{array}{c}-0,98 \\
(-1,55) \\
0,33 \\
48\end{array}$ & $\begin{array}{c}-0,39 * * \\
(-2,14) \\
0,25 \\
48\end{array}$ \\
\hline Colombia & $\begin{array}{c}0,45 \\
(0,73) \\
0,58 \\
52\end{array}$ & $\begin{array}{c}0,04 \\
(0,18) \\
0,22 \\
49\end{array}$ & $\begin{array}{c}0,67 \\
(1,05) \\
0,59 \\
48\end{array}$ & $\begin{array}{c}0,04 \\
(0,18) \\
0,22 \\
46\end{array}$ \\
\hline México & $\begin{array}{c}-0,55 \\
(-0,78) \\
0,14 \\
61\end{array}$ & $\begin{array}{c}-0,62 * * \\
(-2,59) \\
0,08 \\
58\end{array}$ & $\begin{array}{c}-1,93 * * \\
(-2,67) \\
0,30 \\
48\end{array}$ & $\begin{array}{c}-0,81^{*} \\
(-3,17) \\
0,15 \\
48\end{array}$ \\
\hline Perú & $\begin{array}{c}1,01 \\
(1,17) \\
0,27 \\
61\end{array}$ & $\begin{array}{c}-1,09 * \\
(-7,12) \\
0,46 \\
58\end{array}$ & $\begin{array}{c}2,45^{*} \\
(3,88) \\
0,55 \\
48\end{array}$ & $\begin{array}{c}-1,03 * \\
(-6,15) \\
0,44 \\
48\end{array}$ \\
\hline
\end{tabular}

Fuente: datos de la página web de estadísticas de la Comisión Económica para América Latina y el Caribe (CEPAL).

Datos trimestrales para cada país:

En la primera fila se muestra el valor estimado de $\delta_{i}$.

En la segunda fila se presentan los estadísticos $t$ según la hipótesis de nulidad $H_{0}: \delta_{i}=0$.

Los niveles de significatividad son los siguientes: $1 \%\left({ }^{*}\right)$ y $5 \%(* *)$, respectivamente.

En la tercera y cuarta filas se muestran los datos estadísticos $\bar{R}^{2}$ y el número de observaciones, respectivamente.

PIB: producto interno bruto. 
En la ecuación (2), $x_{i, t}$ representa la variable de interés y $F_{i, t}$ es una variable ficticia que toma el valor 1 para los datos observados de los países que adoptaron MI y el valor 0 para los que no lo hicieron. El parámetro $\delta$ mide la diferencia (en promedio) entre los valores $x_{i, t}$ de los dos grupos de países.

$$
x_{i, t}=\alpha+\delta F_{i, t}+\varepsilon_{i, t}
$$

Se calcularon regresiones para las tres variables de interés - la tasa de inflación, la tasa de interés de los depósitos bancarios y el crecimiento del PIB- sobre la base de datos mensuales y trimestrales del período comprendido entre enero de 2000 y diciembre de 2007. Las variables se definen y se miden del mismo modo que en la sección II, número 2, letra a).

En el cuadro 10 se muestran los resultados de todas las regresiones. Como resalta, los valores estimados de $\delta$ tienen siempre el signo correcto y son estadísticamente significativos al nivel del $1 \%$, excepto en lo referente al crecimiento del PIB. Los resultados relativos a la tasa de inflación (que se presentan en las dos primeras columnas) indican que los países que implantaron MI tuvieron una tasa media de inflación inferior en 4,95 puntos porcentuales con respecto a la de los países que no aplicaron ese régimen. El grupo que implementó MI también obtuvo mejores resultados en cuanto a la variabilidad de la tasa de inflación: la desviación típica se redujo 1,71 puntos porcentuales, en promedio, con respecto a los países que no adoptaron ese régimen. En lo referente a la tasa de interés nominal (en las columnas tercera y cuarta), las mejoras logradas con el esquema de MI se reflejan en la reducción de los niveles y variabilidad de esta variable.

Los resultados relacionados con el crecimiento del PIB merecen comentarios adicionales. En primer lugar, se obtienen sobre la base de datos trimestrales (480 observaciones). Segundo, aunque el signo del parámetro que mide el impacto es correcto, el valor estimado es muy bajo y estadísticamente no significativo, pues el estadístico $t$ vale 0,30. Tercero, la adopción de MI ayudó a reducir la variabilidad del crecimiento del PIB, ya que su parámetro estimado tiene signo negativo y es estadísticamente significativo al 1\%. La fijación de MI contribuyó a aminorar la variabilidad de la tasa de crecimiento en 0,37 puntos porcentuales en promedio con respecto a las economías que no adoptaron este esquema de política monetaria. Estas estimaciones confirman plenamente los resultados obtenidos en el análisis de regresión con series cronológicas, pues se detecta que los países con MI lograron tasas de crecimiento menos volátiles, aunque no necesariamente más elevadas.

\section{Efectos de tratamiento}

Las pruebas econométricas aplicadas hasta la fecha para evaluar la influencia de las variables cualitativas, tales como la adopción de MI, pueden estar sujetas a problemas

\begin{tabular}{|c|c|c|c|c|c|c|}
\hline & \multicolumn{2}{|c|}{ 2000: ene - 2007: dic } & \multicolumn{2}{|c|}{ 2000: ene - 2007: dic } & \multicolumn{2}{|c|}{ 1er. trim. $2000-4^{\circ}$ trim. 2007} \\
\hline & \multicolumn{2}{|c|}{$\begin{array}{l}\text { Tasa de inflación } \\
\text { (datos mensuales) }\end{array}$} & \multicolumn{2}{|c|}{$\begin{array}{l}\text { Tasa de interés } \\
\text { (datos mensuales) }\end{array}$} & \multicolumn{2}{|c|}{$\begin{array}{l}\text { Crecimiento del PIB } \\
\text { (datos trimestrales) }\end{array}$} \\
\hline & Nivel & Desviación típica & Nivel & Desviación típica & Nivel & Desviación típica \\
\hline Metas de inflación & $\begin{array}{c}-4,95 \\
(-7,63)^{*} \\
1440 \\
0,04\end{array}$ & $\begin{array}{c}-1,71 \\
(-8,93)^{*} \\
1440 \\
0,05\end{array}$ & $\begin{array}{c}-2,60 \\
(-5,14)^{*} \\
1440 \\
0,02\end{array}$ & $\begin{array}{c}-1,20 \\
(-6,39)^{*} \\
1440 \\
0,03\end{array}$ & $\begin{array}{l}0,12 \\
(0,30) \\
480 \\
-0,02\end{array}$ & $\begin{array}{c}-0,37 \\
(-3,19)^{*} \\
480 \\
0,02\end{array}$ \\
\hline
\end{tabular}

Fuente: datos de la página web de estadísticas de la Comisión Económica para América Latina y el Caribe (CEPAL) y base de datos de Estadísticas Financieras Internacionales del Fondo Monetario Internacional.

Notas: en la primera fila se muestra el valor estimado de $\delta$.

Los números entre paréntesis corresponden a los estadísticos $t$ según la hipótesis de nulidad $H_{0}: \delta=0$.

El nivel de significación es de $1 \%(*)$.

En la tercera y cuarta filas se muestra el número de observaciones de datos de panel realizadas y los estadísticos $\bar{R}^{2}$, respectivamente.

Los países que adoptaron objetivos directos de inflación son: Brasil, Chile, Colombia, México y Perú. El grupo de países que no implantaron ese régimen está compuesto por: Argentina, Bolivia (Estado Plurinacional de), Costa Rica, Ecuador, El Salvador, Panamá, Paraguay, República Dominicana, Uruguay y Venezuela (República Bolivariana de).

PIB: producto interno bruto. 
de endogeneidad. Para superar esta limitación, en esta subsección se estima un modelo de efectos de tratamiento diseñado especialmente para investigar la repercusión de las variables no observables en variables cuantitativas, resolviendo además el sesgo de autoselección. Se aplica el modelo sugerido por Heckman (1979); Maddala (1983), y Greene (2003) para estimar los efectos de las MI en los niveles y variabilidad de las tres variables de interés. El método consta de dos estimaciones secuenciales. Primero, se realiza la estimación de una ecuación probit con el objeto de evaluar en qué medida algunas variables propuestas en la literatura empírica afectan a la probabilidad de adoptar un régimen de MI. A continuación se estiman tres ecuaciones de respuesta que relacionan cada variable de interés con sus determinantes principales, entre los que se encuentra la aplicación de MI. El modelo es el siguiente:

$$
\begin{gathered}
y_{i t}=\beta^{\prime} x_{i t}+\delta I T_{i t}+u_{i t} \\
I T_{i t}= \begin{cases}1 & \text { si } I T_{i t}^{*}>0 \\
0 & \text { en caso contrario }\end{cases} \\
I T_{i t}^{*}=\gamma^{\prime} w_{i t}+\zeta_{i t}
\end{gathered}
$$

La expresión (3) es la ecuación de respuesta (output equation) para cada variable de interés $y_{i t}$. En ella se muestra que $y_{i t}$ (el valor de $y$ en el país $i$ durante el trimestre $t$ ) tiene dos determinantes principales: el vector $x_{i t}$, que incluye un conjunto de variables observables que pueden afectar a $y_{i t}$, y una variable dicotómica, $I T_{i t}$, que toma el valor 1 si el país $i$ aplica metas de inflación en el período $t$, y el valor 0 en caso contrario, tal como indica la expresión (4). En lo que respecta a los componentes del vector $x_{i, t}$, se han seleccionado cuatro variables incluidas en modelos macroeconómicos estándar: formación bruta de capital fijo $\left(g f f k_{i t}\right)$, apertura externa $\left(\right.$ open $\left._{i t}\right)$, tasa de crecimiento de la masa monetaria $\left(m_{1}\right)$ y déficit público como porcentaje del PIB $\left(d e f_{i t}\right)$. El parámetro $\delta$ mide el efecto de la adopción de objetivos de inflación en la variable $y_{i t}$.

En las columnas 2 a 4 del cuadro 11 se describen los signos hipotéticos de los efectos de las variables exógenas en los niveles de las tres variables endógenas de las ecuaciones de respuesta. Con respecto a las variables nominales (inflación y tasas de interés), parece claro que la apertura externa debería provocar una caída de ambos niveles nominales. Además, se considera que con el incremento de la masa monetaria subiría la inflación y bajarían las tasas de interés a corto plazo. Sin embargo, no hay argumentos concluyentes para determinar el signo del déficit fiscal. Si los déficits fiscales se financian sobre todo con deuda, deberían aumentar las tasas de interés y reducir la tasa de inflación. Pero si provocan expansiones monetarias, cabría esperar efectos en sentido contrario. En cuanto a la incidencia en el crecimiento económico, se espera que las dos primeras variables del vector $x_{i t}$ tengan efectos positivos. El efecto favorable de $g f f k_{i t}$ en el crecimiento económico se documenta ampliamente en los modelos tradicionales de crecimiento económico, y la contribución positiva de la apertura externa a la expansión económica ha sido demostrada empíricamente por quienes defienden la hipótesis del crecimiento impulsado por las exportaciones (véase, por ejemplo, Feder, 1983; Helpman y Krugman, 1985; Krugman 1987), así como por la teoría del crecimiento endógeno (Romer, 1986; Grossman y Helpman, 1985;

\begin{tabular}{|c|c|c|c|c|}
\hline & & Ecuación de resultados & & Ecuación probit \\
\hline Variable de control & Tasa de inflación & Tasa de interés de corto plazo & Crecimiento del PIB & \\
\hline$g f f k_{i t}$ & & & + & \\
\hline open $_{i t}$ & - & - & + & - \\
\hline$I T_{i t}$ & - & - & $+1-$ & \\
\hline$m_{l}$ & + & - & & \\
\hline$d e f_{i t}$ & $+/-$ & $+/-$ & & $+/-$ \\
\hline$d e p_{i t}$ & & & & + \\
\hline$c a_{i t}$ & & & & - \\
\hline extdebt $_{i t}$ & & & & - \\
\hline
\end{tabular}

Signos hipotéticos en las ecuaciones de efectos de tratamiento

Fuente: elaboración propia sobre la base de modelos macroeconómicos convencionales.

PIB: producto interno bruto. 
CUADRO 12

Efectos de tratamiento: el efecto de las metas de inflación

(1er. trim. $2000-4^{\circ}$ trim. 2007)

Variable de tratamiento: aplicación de la estrategia de fijación de objetivos directos de inflación ( $\left.I T_{i t}^{*}\right)$. Ecuación probit.

\begin{tabular}{lc}
\hline Variable & $\begin{array}{c}\text { Metas de } \\
\text { inflación }\end{array}$ \\
\hline$c$ & $5,468^{*}$ \\
& $(8,769)$ \\
def $_{i t}$ & $0,142^{*}$ \\
& $(4,305)$ \\
open $_{i t}$ & $-0,064^{*}$ \\
& $(-9,631)$ \\
dep $_{i t}$ & $0,089^{*}$ \\
& $(8,070)$ \\
ca $_{i t}$ & $-0,214^{*}$ \\
& $(-7,051)$ \\
extdebt & $-0,100^{*}$ \\
& $(-8,810)$ \\
\hline
\end{tabular}

\begin{tabular}{|c|c|c|c|c|c|c|}
\hline \multicolumn{7}{|c|}{ Variable de respuesta } \\
\hline Variable & Inflación & $\begin{array}{l}\text { Desviación } \\
\text { típica de la } \\
\text { inflación }\end{array}$ & $\begin{array}{l}\text { Tasa de } \\
\text { interés }\end{array}$ & $\begin{array}{l}\text { Desviación } \\
\text { típica de las } \\
\text { tasas de interés }\end{array}$ & $\begin{array}{l}\text { Crecimiento } \\
\text { del PIB }\end{array}$ & $\begin{array}{l}\text { Desviación típica } \\
\text { del crecimiento } \\
\text { del PIB }\end{array}$ \\
\hline$c$ & $\begin{array}{l}5,045^{* * *} \\
(1,656)\end{array}$ & $\begin{array}{c}0,570 \\
(0,655)\end{array}$ & $\begin{array}{c}22,869^{*} \\
(9,916)\end{array}$ & $\begin{array}{l}2,100 * * \\
(2,517)\end{array}$ & $\begin{array}{c}0,149 \\
(0,148)\end{array}$ & $\begin{array}{l}1,178^{*} \\
(4,117)\end{array}$ \\
\hline gffk $k_{i t}$ & & & & & $\begin{array}{c}0,159^{*} \\
(3,681)\end{array}$ & $\begin{array}{c}-0,014 \\
(-1,012)\end{array}$ \\
\hline open $_{i t}$ & $\begin{array}{l}-0,102^{*} \\
(-6,285)\end{array}$ & $\begin{array}{l}-0,022 * \\
(-4,840)\end{array}$ & $\begin{array}{l}-0,078^{*} \\
(-6,306)\end{array}$ & $\begin{array}{l}-0,027 * \\
(-5,970)\end{array}$ & $\begin{array}{l}0,010 * * * \\
(1,681)\end{array}$ & $\begin{array}{l}-0,013 * \\
(-5,641)\end{array}$ \\
\hline$I T_{i t}$ & $\begin{array}{l}-3,362^{* *} \\
(-1,966)\end{array}$ & $\begin{array}{l}-1,118 * * \\
(-2,289)\end{array}$ & $\begin{array}{c}-7,290 * \\
(-5,633)\end{array}$ & $\begin{array}{l}-1,177 * * \\
(-2,514)\end{array}$ & $\begin{array}{c}0,062 \\
(0,329)\end{array}$ & $\begin{array}{l}-0,236^{* *} \\
(-1,923)\end{array}$ \\
\hline$\hat{\lambda}_{i t}$ & $\begin{array}{c}17,188^{*} \\
(3,527)\end{array}$ & $\begin{array}{c}4,110^{*} \\
(2,951)\end{array}$ & $\begin{array}{l}-6,964 * * * \\
(-1,888)\end{array}$ & $\begin{array}{c}1,918 \\
(1,437)\end{array}$ & $\begin{array}{c}-0,037 \\
(-0,139)\end{array}$ & $\begin{array}{c}1,709 * \\
(3,635)\end{array}$ \\
\hline$m_{l}$ & $\begin{array}{l}0,088 * * \\
(2,257)\end{array}$ & $\begin{array}{c}0,049^{*} \\
(4,383)\end{array}$ & $\begin{array}{l}-0,083^{*} \\
(-2,821)\end{array}$ & $\begin{array}{c}0,017 \\
(1,570)\end{array}$ & & \\
\hline$d e f_{i t}$ & $\begin{array}{l}0,297 * * * \\
(1,613)\end{array}$ & $\begin{array}{c}0,152^{*} \\
(2,882)\end{array}$ & $\begin{array}{l}-0,325^{* *} \\
(-2,330)\end{array}$ & $\begin{array}{l}-0,102 * * \\
(-2,023)\end{array}$ & & \\
\hline Observaciones & 480 & 480 & 480 & 480 & 480 & 480 \\
\hline
\end{tabular}

Fuente: datos de la página web de estadísticas de la Comisión Económica para América Latina y el Caribe (CEPAL) y base de datos de Estadísticas Financieras Internacionales del Fondo Monetario Internacional.

Notas:

Niveles de significatividad: $1 \%(*), 5 \%(* *)$ y $10 \%(* * *)$.

Los valores entre paréntesis corresponden a los cuartiles de la distribución normal típica (estimación probit) y a los valores de los estadísticos $t$ (estimaciones de las ecuaciones de respuesta ).

PIB: producto interno bruto. 
Alesina y Rodrik, 1999). Por otra parte, Hassan (2005) y Ekanayake, Vogel y Veeramacheneni (2003) ofrecen evidencia empírica de los efectos positivos del comercio internacional en el crecimiento económico de algunos grupos de economías de mercado emergentes.

La expresión (5) es la ecuación probit, estableciendo que la probabilidad de adoptar MI, representada mediante una variable no observada, $I T_{i t}^{*}$, depende a su vez de un conjunto de factores incluidos en el vector $w_{i t}$. Tras examinar los determinantes potenciales de la fijación de MI sugeridos con más frecuencia en la bibliografía — Gerlach (1999); Hu (2006); Batini y Laxton (2007); Leyva (2008) — se han seleccionado las siguientes variables: i) déficit fiscal; ii) apertura externa; iii) vigor del desarrollo financiero; iv) vulnerabilidad a fluctuaciones externas, y v) nivel de endeudamiento externo expresado en dólares.

Se explicará ahora cómo se miden las variables incluidas en la ecuación probit, así como el signo esperado de la influencia en la probabilidad de adoptar MI (véase la cuarta columna del cuadro 12). El déficit fiscal se mide como porcentaje del PIB y, tal como en el caso de la ecuación de respuesta, no hay argumentos definitivos para formular una hipótesis sobre el signo de esta variable. Por una parte, la independencia del banco central (ingrediente importante de los regímenes de MI) obliga a los gobiernos a aplicar políticas fiscales más austeras. Pero, por otra, la implementación de MI podría decidirse endógenamente como un remedio para las prácticas presupuestarias públicas menos consistentes. En consecuencia, puede esperarse cualquiera de los dos signos. La apertura externa se mide por la suma de importaciones y exportaciones como proporción del PIB. Debido a que las variaciones de los tipos de cambio se trasladan en gran parte a los precios internos, la apertura interfiere en el control de la inflación y disuade al banco central de fijar objetivos explícitos de inflación. Esto ocurre especialmente en economías de mercado emergentes por tener coeficientes de traslación más elevados que los de los países industrializados (García-Solanes y Torrejón-Flores, 2010). Además, como se destaca en Mishkin (2001); Eichengreen (2002), y Willett (2002), una mayor apertura comercial y financiera en un marco de tipos de cambio flexibles puede originar inestabilidad financiera en economías de mercado emergentes con MI. Por todo ello, es razonable esperar que exista una relación negativa entre la apertura y la probabilidad de adoptar dichas metas.

El grado de solvencia y desarrollo de un sistema financiero se puede representar aproximadamente por el total de depósitos bancarios como proporción del
PIB. Puesto que esta variable es un elemento clave - si no un requisito- de una estrategia de fijación de objetivos explícitos de inflación, se puede suponer que contribuye a la adopción de un régimen de este tipo. Consecuentemente, el signo debe ser positivo. La vulnerabilidad a las perturbaciones externas se mide mediante el déficit de la cuenta corriente. Dado que cuando existen grandes desequilibrios externos el banco central se ve menos inclinado a implementar MI, el signo de esta variable debería ser negativo. Por último, las obligaciones externas en divisas se miden con la acumulación (stock) de la deuda externa en moneda extranjera. Se deduce fácilmente que, dado que el alto nivel de la deuda va en detrimento de la flexibilidad de los tipos de cambio, las obligaciones externas disminuyen el margen de maniobra para adoptar objetivos explícitos de inflación ${ }^{3}$. Por consiguiente, se supone que el signo será negativo.

Para la estimación del sistema de ecuaciones (3) a (5), se puede aplicar el método de máxima verosimilitud, o bien el procedimiento en dos etapas sugerido por Heckman (1979), explicado detalladamente por Maddala (1983) y Greene (2003) y utilizado por Edwards (2004). En este trabajo se escogió la metodología de dos etapas, cuyo significado se explica brevemente a continuación. En la primera etapa se aplicó el método probit para la estimación del parámetro $\gamma$, que luego se utiliza en la ecuación (5) para calcular la variable $I T_{i t}^{*}$. El resultado se aplica en la ecuación (4) para obtener la variable dicotómica $I T_{i t}$. En la segunda etapa se realizó la estimación de las ecuaciones de respuesta. No obstante, para obtener un valor consistente y no sobrestimado del parámetro $\delta$ en la ecuación (3), se debe incorporar en esa ecuación la variable $\hat{\lambda}_{i t}$, que se define como la relación entre la función de densidad y la distribución acumulada de $I T_{i t}^{*}: \hat{\lambda}_{i t}=f\left(I \hat{T}_{i t}^{*}\right) / F\left(I \hat{T}_{i t}^{*}\right)$ (véase Greene, 2003, cap. 22). Por consiguiente, se realiza una estimación de la ecuación ampliada, $y_{i t}=\beta^{\prime} x_{i t}+\delta I T_{i t}+\beta_{\hat{\lambda}} \hat{\lambda}_{i t}+\xi_{i t}$, para estimar los valores de los parámetros $\beta, \beta_{\hat{\lambda}}$ y $\delta$.

En el cuadro 12 se presentan los resultados estimados del sistema de ecuaciones (3) a (5) en relación con las tres variables de interés. En lo referente a la ecuación probit, todas las variables seleccionadas como determinantes de la probabilidad de implantar MI presentan el signo hipotético correcto y son estadísticamente significativas al nivel del $1 \%$, lo que entraña que la probabilidad de

\footnotetext{
${ }^{3}$ Aunque la dolarización de la deuda obstaculiza claramente la implantación de un sistema de objetivos de inflación, por sí sola no impide la posibilidad de usar ese esquema como un régimen de política monetaria. El caso de la economía peruana — muy dolarizada - es un buen ejemplo, como muestran Leiderman, Maino y Parrado (2006).
} 
adoptar MI queda bien reflejada por las variables seleccionadas y se ve favorecida si concurren los siguientes factores: i) desequilibrios fiscales; ii) escasa apertura económica; iii) solidez del sistema financiero nacional; iv) déficits de cuenta corriente reducidos, y v) bajo nivel de deuda externa.

En cuanto a las ecuaciones de respuesta, todas las estimaciones se caracterizan por una alta calidad estadística, aunque presentan grandes diferencias. Así, el nivel y la variabilidad de la inflación y las tasas de interés se explican fácilmente por la apertura externa, la expansión de la masa monetaria, el déficit público y la fijación de MI. Todos estos determinantes tienen los signos hipotéticos que figuran en el cuadro 11. Cabe destacar que la expansión de la masa monetaria afecta considerablemente al nivel de las tasas de interés, pero no a su volatilidad. La constatación más relevante es que la implementación de MI tiene repercusiones claramente negativas tanto en el nivel como en la volatilidad de la inflación y las tasas de interés, con lo que se confirman los resultados de las secciones anteriores. Si se observa ahora la ecuación del crecimiento, los resultados presentados en las columnas quinta y sexta de la sección inferior del cuadro 12 indican que el crecimiento económico se ve favorecido por la formación bruta de capital fijo (FBCF) y por la apertura internacional. La adopción de MI contribuye significativamente a moderar la variabilidad del crecimiento del PIB, pero no tiene una gran incidencia en el nivel de esa variable, lo que nuevamente confirma las observaciones ya presentadas en este artículo. Este resultado no parece sorprendente, dado que los efectos de la aplicación de MI en el crecimiento deberían revelarse en horizontes cronológicos más largos.

\section{IV}

\section{Observaciones finales}

En este artículo se ha analizado hasta qué punto la adopción de objetivos de inflación ha mejorado el desempeño macroeconómico de cinco países de América Latina que implantaron este tipo de régimen de política monetaria. Para evaluar los resultados en términos relativos, se ha tomado como referencia a un grupo de 10 países de América Latina que comparten muchas características institucionales con las cinco economías objeto de estudio, pero en las que no se ha implementado un sistema de MI. Es de esperar que la similitud geográfica e institucional permita extraer conclusiones más claras sobre los efectos específicos de la adopción de objetivos de inflación.

Para tener una idea aproximada de los resultados que un régimen de MI podría producir tanto en los niveles como en la variabilidad de algunas variables dentro de cada país, en la sección II se realizó un análisis descriptivo y se aportaron pruebas estadísticas a partir de datos relativos a los 15 países de América Latina involucrados en el estudio. En la sección III se aplicaron tres tipos de contrastes econométricos distintos a fin de evaluar con mayor precisión el efecto de la adopción de MI. En los dos primeros análisis econométricos, basados en series cronológicas y regresiones con datos de panel, se utilizaron variables ficticias para evaluar de forma indirecta los efectos del esquema de MI en algunas variables macroeconómicas. Dado que los resultados de estas pruebas convencionales pueden verse afectados por problemas de endogeneidad y autoselección, se realizó la estimación de un modelo de efectos de tratamiento especialmente indicado para evaluar la repercusión de las variables cualitativas (como la implementación de MI) en otras variables fáciles de cuantificar (como la inflación, las tasas de interés y el crecimiento del PIB). Esta es la principal contribución técnica original del artículo a la bibliografía disponible sobre el tema. Los resultados de las tres pruebas econométricas confirman las impresiones descriptivas, al indicar que la fijación de MI es responsable de la reducción de la variabilidad y de los niveles de la inflación y de las tasas de interés a corto plazo, así como de una menor variabilidad del crecimiento en países que adoptaron este régimen. Sin embargo, ninguna de las tres pruebas econométricas permitió aclarar cuáles fueron los efectos de la aplicación de MI en el crecimiento del PIB. En resumen, se ofrece evidencia de que la implantación del régimen de MI en los cinco países de América Latina contribuyó a mejorar el desempeño económico durante el período investigado, probablemente como consecuencia de una disminución de las expectativas de inflación causada por ese régimen en esos países. 
Alesina, A. y D. Rodrik (1999), "Distributive politics and economic growth", Quarterly Journal of Economics, vol. 109, No 2 , Cambridge, Massachusetts, The MIт Press.

Ball, L. y N. Sheridan (2005), "Does inflation targeting matter?", The Inflation Targeting Debate, B.S. Bernanke y M. Woodford (eds.), Chicago, University of Chicago Press.

Batini, N. y D. Laxton (2007), "Under what conditions can inflation targeting be adopted? The experience of emerging markets", Monetary Policy under Inflation Targeting, F. Mishkin y K. SchmidtHebbel (eds.), Santiago de Chile, Banco Central de Chile.

Berg, A., E. Borensztein y P. Mauro (2002), "An evaluation of monetary regime options for Latin America", IMF Working Paper, $\mathrm{N}^{\circ}$ 02/211, Washington, D.C., Fondo Monetario Internacional.

Blanchard, O. (2005), "Fiscal dominance and inflation targeting: Lessons from Brazil", Inflation Targeting, Debt, and the Brazilian Experience, 1999 to 2003, F. Giavazzi, I. Goldfajn y S. Herrera (eds.), Cambridge, Massachusetts, The MIT Press.

Blejer, M.I. y otros (2001), "Inflation targeting in the context of IMFsupported adjustment programs", IMF Working Paper, $\mathrm{N}^{\mathrm{o}}$ 01/31, Washington, D.C., Fondo Monetario Internacional.

Brito, R.D. y B. Bystedt (2010), "Inflation targeting in emerging economies: Panel evidence", Journal of Development Economics, vol. 91, $\mathrm{N}^{\circ} 2$, Amsterdam, Elsevier.

Calderón, C. y K. Schmidt-Hebbel (2003a), "Learning the hard way: Ten lessons for Latin America's turmoil”, Santiago de Chile, Banco Central de Chile, inédito.

(2003b), "Macroeconomic policies and performance in Latin America", Documento de trabajo, № 217, Santiago de Chile, Banco Central de Chile.

Calvo, G.A. y F. Mishkin (2003), "The mirage of exchange rate regimes for emerging market countries", Journal of Economic Perspectives, vol. 17, № 4, Nashville, Tennessee, American Economic Association.

Calvo, G.A. y C. Reinhart (2002), "Fear of floating", Quarterly Journal of Economics, vol. 117, No 2, Cambridge, Massachusetts, The MIT Press.

Corbo, V., O. Landerretche y K. Schmidt-Hebbel (2002), "Does inflation targeting make a difference", Inflation Targeting: Design, Performance, Challenges, N. Loayza y R. Soto (eds.), Santiago de Chile, Banco Central de Chile.

Edwards, S. (2004), "Thirty years of current account imbalances, current account reversals and sudden stops", IMF Staff Papers, vol. 51, número especial, Washington, D.C., Fondo Monetario Internacional.

Edwards, S. y E. Levy-Yeyati (2003), "Flexible exchange rates as shock absorbers", European Economic Review, vol. 49, No 8, Amsterdam, Elsevier, noviembre.

Eichengreen, B. (2002), "Can emerging markets float? Should they inflation target?”, Working Paper Series, N 36, Brasilia, Banco Central del Brasil, febrero.

Ekanayake, E.M., R. Vogel y B. Veeramacheneni (2003), "Openness and economic growth: Empirical evidence on the relationship between output, inward FDI, and trade", Journal of Business Strategies, número de primavera.

Feder, G. (1983), "On exports and economic growth", Journal of Development Economics, vol. 12, № 1-2, Amsterdam, Elsevier.

FMI (Fondo Monetario Internacional) (2006), Inflation Targeting and the IMF, Washington, D.C., marzo.

(2005), "Régimen de metas de inflación: ¿Es viable en los países de mercados emergentes?", Perspectivas de la economía mundial, Washington, D.C., septiembre.

García-Solanes, J. y F. Torrejón-Flores (2010), "Devaluation and passthrough in indebted and risky economies", International Review of Economics and Finance, vol. 19, № 1, Amsterdam, Elsevier.

Gerlach, S. (1999), "Who targets inflation explicitly?", European Economic Review, vol. 43, № 7, Amsterdam, Elsevier.
Gonçalves, C.E.S y J.M. Salles (2008), "Inflation targeting in emerging economies: What do the data say?", Journal of Development Economics, vol. 85, № 1-2, Amsterdam, Elsevier.

Greene, W. (2003), Econometric Analysis, New Jersey, Prentice Hall. Grosssman, G. y E. Helpman (1985), “Technology and trade", Handbook of International Economics, G. Grossman y K. Rogoff (eds.), vol. 3, Amsterdam, Elsevier.

Hassan, A.F.M.K. (2005), "Trade openness and economic growth: search for a causal relationship", South Asian Journal of Management, vol. 12, $\mathrm{N}^{\circ} 4$, Hyderabad, AMDISA, octubre.

Heckman, J. (1979), "Sample selection bias as a specification error", Econometrica, vol. 47, № 1, Nueva York, Econometric Society, enero.

Helpman, E. y P.R. Krugman (1985), Market Structures and Foreign Trade, Cambridge, Massachusetts, The MIT Press.

Hrncir, M. y K. Smidkova (2003), "The Czech approach to inflation targeting", Macroeconomics, N $\mathrm{N}^{\circ}$ 0303019, EconWPA.

$\mathrm{Hu}, \mathrm{Y}$. (2006), "The choice of inflation targeting - an empirical investigation", International Economics and Economic Policy, vol. $3, \mathrm{~N}^{\mathrm{o}} 1$, Springer.

Jonas, J. y F. Mishkin (2005), "Inflation targeting in transition economies: Some issues and experience", The Inflation-Targeting Debate, B. Bernanke y M. Woodford (eds.), Chicago, The University of Chicago Press.

King, M. (2005), Monetary Policy: Practice Ahead of Theory. The Mais Lecture 2005: Speech by the Governor, Londres, Cass Business School, City University, 17 de mayo.

Krugman, P.R. (1987), The Age of Diminished Expectation, Cambridge, Massachusetts, The MIT Press.

Kvasnicka, M. (2000), "Inflation targeting. Is it suitable for transitional economies?", IV International Conference of Doctoral Students, vol. 1, Brno, Brno University of Technology.

Leiderman, L., R. Maino y E. Parrado (2006), "Inflation targeting in dollarized economies", Documento de trabajo, $\mathrm{N}^{\circ} 368$, Santiago de Chile, Banco Central de Chile.

Leyva, G. (2008), "The choice of inflation targeting", Documento de trabajo, $\mathrm{N}^{\circ} 475$, Santiago de Chile, Banco Central de Chile.

Lin, S. y H. Ye (2009), "Does inflation targeting make a difference in developing countries?", Journal of Development Economics, vol. 89, No 1, Amsterdam, Elsevier.

Maddala, G.S. (1983), Limited-Dependent and Qualitative Variables in Econometrics, Nueva York, Cambridge University Press.

Mishkin, F.S. (2001), "Global financial instability: Framework, events, issues", Journal of Economic Perspectives, vol. 13, $\mathrm{N}^{\mathrm{o}} 4$, Nashville, Tennessee, American Economic Association. (1999), "International experiences with different monetary policy regimes", Journal of Monetary Economics, vol. 43, No 3, Amsterdam, Elsevier.

Mishkin, F.S. y M. Savastano (2002), "Monetary policy strategies for emerging market countries: Lessons from Latin America", Comparative Economics Studies, vol. 44, No 2-3, Palgrave Macmillan.

Mishkin, F. y K. Schmidt-Hebbel (2007), "Does inflation targeting make a difference?", Monetary Policy Under Inflation Targeting, F. Mishkin y K. Schmidt-Hebbel (eds.), Santiago de Chile, Banco Central de Chile.

Nogueira, R.P. y M.A. León-Ledesma (2009), "Fear of floating in Brazil: Did inflation targeting matter?", North American Journal of Economics and Finance, vol. 20, № 3, Amsterdam, Elsevier.

Romer, P.M. (1986), "Increasing returns and long run growth", Journal of Political Economy, vol. 94, No 5, Chicago, University of Chicago Press.

Sims, C. (2005), "Limits to inflation targeting", The Inflation Targeting Debate, B. Bernanke y M. Woodford (eds.), Chicago, The University of Chicago Press. 
Svensson, L. (1997), "Inflation forecast targeting: implementing and monitoring inflation targets", European Economic Review, vol. 41, $\mathrm{N}^{\circ} 6$, Amsterdam, Elsevier.

Valdés, R. (2007), "Success of a flexible IT regime in reducing inflation in Chile", Documentos de política económica, $\mathrm{N}^{\circ} 22$, Santiago de Chile, Banco Central de Chile, noviembre.
Willett, T.D. (2002), "Fear of floating needn't imply fixed rates: feasible options for intermediate exchange rate regimes", mayo, inédito.

Yigit, T.M. (2010), "Inflation targeting: An indirect approach to assess the direct impact", Journal of International Money and Finance, vol. 29, $\mathrm{N}^{\circ}$ 7, Amsterdam, Elsevier. 